\title{
Histological Examination of Collagen and Proteoglycan Changes in Osteoarthritic Menisci
}

\author{
Yubo Sun ${ }^{*}, 1$, David R. Mauerhan ${ }^{1}$, Jeffrey S. Kneisl ${ }^{1}$, H. James Norton ${ }^{2}$, Natalia Zinchenko ${ }^{1}$, \\ Jane Ingram ${ }^{1}$, Edward N. Hanley Jr. ${ }^{1}$ and Helen E. Gruber ${ }^{1}$ \\ ${ }^{I}$ Department of Orthopedic Surgery, ${ }^{2}$ Department of Biostatistics, Carolinas Medical Center, PO Box 32861, Charlotte,
NC 28232, USA
}

\begin{abstract}
This study sought to examine collagen and proteoglycan changes in the menisci of patients with osteoarthritis (OA). Collagens were examined using picrosirius red, and hematoxylin and eosin. Proteoglycans were examined using safranin-O and alcian blue. Types I and II collagens and aggrecan were examined using immunochemistry. Severe loss of collagens was observed to occur in OA menisci, particularly in the middle and deep zones and collagen networks were less organized than those of normal menisci. In contrast, proteoglycan staining in the middle and deep zones of OA meniscus increased compared to normal control menisci. Immunohistochemistry indicated that types I and II collagens were co-localized and the loss of types I collagen in OA menisci appeared more severe in the middle and deep zones than that in the surface zones. The loss of type II collagen however was severe across all three zones. Immunohistochemistry also indicated elevated aggrecan staining in OA menisci. These findings together indicate that severe loss of collagens and intrameniscal degeneration are hallmarks of OA menisci and that extracellular matrix degeneration occurred in OA menisci follows a pathway different from that occurred in OA articular cartilage. These findings are not only important for a better understanding of the disease process but also important for the development of novel structure-modifying drugs for OA therapy.
\end{abstract}

Keywords: Aggrecan, collagen I, collagen II, meniscus, osteoarthritis, proteoglycan.

\section{INTRODUCTION}

Osteoarthritis (OA) is not merely an articular cartilage disease, but a disease of the whole joint involving synovium, subchondral bone and meniscus. The knee menisci are specialized tissues that play a vital role in load transmission, shock absorption and joint stability. An accumulation of recent evidence suggests that knee menisci may not be passive bystanders in the disease process of OA. The coincidence of horizontal cleavage lesions of meniscus and $\mathrm{OA}$ is frequent [1]. The prevalence of meniscal tears is $63 \%$ among patients with radiographic evidence of OA, but only $23 \%$ among patients without radiographic evidence of OA [2]. Several MRI (Magnetic Resonance Imaging) studies revealed that meniscal degeneration was a general feature of $\mathrm{OA}$ and that meniscal degenerative lesions were a potent risk factor for the development of OA [3-7].

Several groups recently reported that the grades of meniscal degenerative tears were correlated with the severity of cartilage lesions in OA [8-10]. We reported that meniscal degeneration in patients with end-stage OA were correlated with the severity of articular cartilage degeneration and that OA meniscal cells produced more calcium deposits than normal meniscal cells $[11,12]$. Moreover, we found that OA meniscal cells expressed aggrecan at a significantly higher level than normal meniscal cells [12]. These findings suggest

*Address correspondence to this author at the Cannon Research 303, Department of Orthopedic Surgery, Carolinas Medical Center, PO Box 32861, Charlotte, NC 28232, USA; Tel: 704/355-5656; Fax: 704/355-5620;

E-mail: yubo.sun@carolinashealthcare.org that the knee menisci may be actively involved in the disease process of OA.

Articular cartilage degeneration in OA has been studied extensively. It has been demonstrated that collagens in OA articular cartilage display much less organized networks and that collagen content falls in advanced OA compared to early OA [13-15]. Proteoglycan content also falls in OA articular cartilage. However, our review of the literatures shows that most previous examinations of OA menisci are performed using animal models of $\mathrm{OA}$ and that there are few prior examinations of collagen and proteoglycan changes in human OA menisci. In contrast to articular cartilage, which mainly contains type II collagen and abundance of proteoglycans, meniscus contains approximately $98 \%$ type I collagen, and less than $1 \%$ of proteoglycans. Therefore, it is likely that the biochemical changes in OA menisci and OA articular cartilage follow different patterns. We decided to perform this study to determine the collagen and proteoglycan changes in human OA menisci to have a better understanding of the disease process, which is important for the development of structure-modifying drugs that target meniscal degeneration for OA therapy.

\section{MATERIALS AND METHODOLOGY}

\section{Materials}

Superfrost-Plus microscope slides and neutral buffered formalin $(10 \%)$ were obtained from Allegiance Inc (McGaw Park, IL). Paraplast Plus paraffin was obtained from Thermo Shandon (Pittsburgh, PA). Safranin-O and fast green were obtained from Polysciences, Inc. (Warrington, PA). 
Picrosirius red, alcian blue, toluidine blue, hematoxylin and eosin were obtained from Sigma (St. Louis, MO). Antibodies specific to type I and type II collagens were obtained from Biodesign International (Kennebunk, ME). Antibody specific to aggrecan was obtained from Santa Cruz Biotechnology (Santa Cruz, CA). Biotinylated second antibody, streptavidin conjugated with horseradish peroxidase and $\mathrm{H}_{2} \mathrm{O}_{2}$ were obtained from Biocare medical (Concord, CA).

\section{Meniscal Specimens}

Meniscal specimens were collected, with the approval of the authors' Institutional Review Board, from eight consecutive unselected OA patients who underwent total joint replacement surgery and from three osteosarcoma patients who underwent lower limb amputation surgery at our medical center. Since these tissues were surgical waste from routine joint replacement surgery and lower limb amputation surgery, and private patient information was not being collected, the need for informed consent was waived.

\section{Histological Analyses of Collagens}

Medial menisci were fixed in $10 \%$ neutral buffered formalin for twenty-four hours and transferred to $70 \%$ ethyl alcohol solution. A portion $4 \mathrm{~mm}$ wide of specimen was transversely excised from the middle part of the meniscus (Fig. 1), embedded in Paraplast Plus paraffin and sectioned with a Leica RM2025 microtome (Nussloch, Germany) to obtain $4 \mu \mathrm{m}$ serial transverse sections of the meniscus. Another portion $15 \mathrm{~mm}$ wide of specimen was again transversely excised from the middle part of the meniscus (Fig. 1). This specimen was divided at the central level horizontally into two pieces. The lower piece was embedded in paraffin and sectioned to obtain $4 \mu \mathrm{m}$ serial horizontal sections of the meniscus.

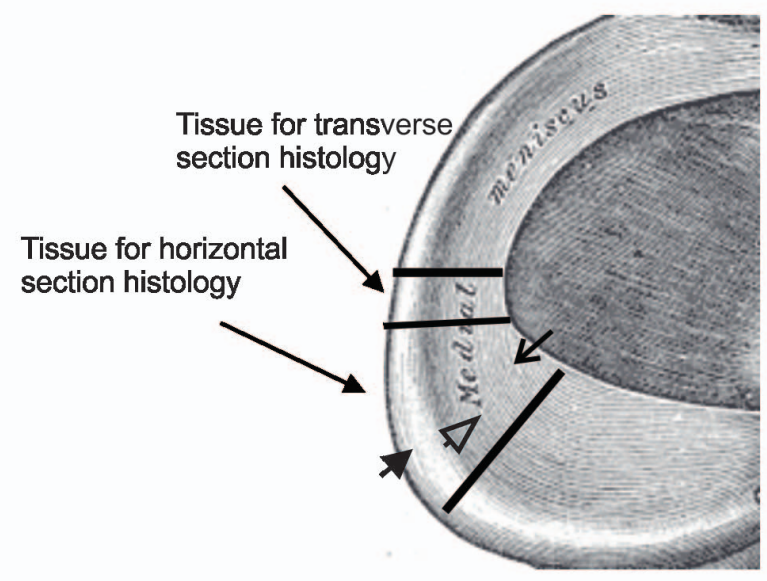

Fig. (1). Harvest sites of medial meniscal specimens. Long line arrows indicate the sites of meniscal specimens harvested for examinations. Short solid arrow indicates the outer portion, short line arrow indicates the inner portion, and short open arrow indicates the central portion of the meniscus.

Sections were mounted on slides and stained with picrosirius red. The results were graded on a scale of 0 to 2 by two independent observers in a blinded manner, where 0 = weak red staining, $1=$ moderate red staining, and $2=$ strong red staining. Additional sections were examined with hematoxylin and eosin staining (H\&E).

\section{Histological Analyses of Proteoglycans}

Sections were stained with safranin-O and fast green. The results were graded on a scale of 0 to 5 by two independent observers in a blinded manner, where $0=$ minimal orangered staining, 1 = very weak orange-red staining, 2 = weak orange-red staining, $3=$ moderate orange-red staining, $4=$ strong orange-red staining, and $5=$ very strong orange-red staining.

Additional sections were examined with alcian blue staining. Results were also graded on a scale of 0 to 5 , where $0=$ minimal blue staining, $1=$ very weak blue staining, $2=$ weak blue staining, $3=$ moderate blue staining, $4=$ strong blue staining, and $5=$ very strong blue staining. Additional sections were also examined with toluidine blue staining.

\section{Immunolocalization of Type I Collagen, type II Collagen and Aggrecan}

Paraffin sections were treated with $3 \% \mathrm{H}_{2} \mathrm{O}_{2}$. The sections were incubated with primary antibody (Final concentration of the antibodies was $2 \mu \mathrm{g} / \mathrm{ml}$ ) specific to type I collagen, type II collagen and aggrecan respectively for 1 hour, and then incubated with a biotinylated second antibody followed with streptavidin conjugated with horseradish peroxidase for 1 hour. Control was performed using mouse serum to replace the primary antibody. Slides were rinsed in phosphate buffered saline three times and visualized using peroxidase substrate diaminobenzidine tetrahydrochloride and $0.02 \% \mathrm{H}_{2} \mathrm{O}_{2}$. Slides were counterstained with light green, dehydrated, cleared and mounted with resinous mounting media.

The immunolocalization of type I collagen in the surface zone and the deep zone of the inner portion of the meniscus was graded by two independent observers in a blinded manner using two different scales. Specifically, the immunolocalization of type I collagen in the surface zone of the menisci was graded on a scale of $0-2$, where $0=$ moderate staining; $1=$ strong staining; and $2=$ very strong staining. The immunolocalization of type I collagen in the deep zone of the menisci was graded on a scale of $0-3$, where $0=$ weak staining; $1=$ moderate staining; $2=$ strong staining; and 3 = very strong staining.

The immunolocalization of type II collagen in the surface and deep zones of inner portion of the menisci was graded by two independent observers in a blinded manner on a scale of $0-4$, where $0=$ very weak staining; $1=$ weak staining; $2=$ moderate staining; $3=$ strong staining, and $4=$ very strong staining. The immunolocalization of aggrecan was graded on a scale of $0-4$, where $0=$ minimal staining; $1=$ weak staining; $2=$ moderate staining, $3=$ wide-spread moderate staining, and $4=$ strong staining.

\section{Statistical Analyses}

The difference of the grades between OA group versus control group was analyzed using the Wilcoxon rank-sum test. In all cases, two-tailed $P<0.05$ was considered significant. Statistical analysis was performed using the $\mathrm{SAS}^{\circledR}$ software, version 9.2 (SAS Institute Inc, Cary, NC). 


\section{RESULTS}

\section{Picrosirius Red Staining}

Representative images of picrosirius red staining are shown in Fig. (2). Normal menisci had strong picrosirius red staining (top photos). In contrast, OA menisci had moderate or weak picrosirius red staining (bottom photos). These results indicated higher collagen content in the normal menisci, and lower collagen content in the OA menisci. The loss of collagen content in OA meniscus occurred across the entire meniscus, indicating the loss of collagens occurred not only in the surface zone of OA meniscus, but also inside OA meniscus (Fig. 2). The grades of picrosirius red staining, along with the demographic patient information, are listed in Table 1. The difference between the intensity of picrosirius red staining in $\mathrm{OA}$ and normal menisci is statistically significant $(\mathrm{P}=0.011)$.

Collagens networks in OA menisci appeared less orderly compared to collagens networks in normal menisci (Fig. 2). Normal control menisci showed well-organized, parallelarranged and compact collagen bundles. In contract, OA menisci showed less organized and less compact collagen networks. To further examine this phenomenon, we performed H\&E staining. Similarly, H\&E staining showed that normal control meniscus had well-organized, parallelarranged and compact collagen bundles with meniscal cells lining up between the collagen bundles (Fig. 3, top photos); whereas that OA meniscus had less organized and less compact collagen networks. In some areas, meniscal cells were lined up between the collagen bundles, but in many other areas, meniscal cells were randomly distributed within OA meniscus (Fig. 3, bottom photos).

\section{Immunolocalization of Type I and Type II Collagens}

Representative images of immunolocalization of type I collagen in the inner portion of normal and OA menisci are shown in Fig. (4). The results indicated that the surface zone (black arrow) of normal meniscus had the highest type I collagen content, and that the type I collagen content was decreased gradually from the surface zone (black arrow) to the middle zone (green arrow) and deep zone (red arrow) (Fig. 4, top photos).

\section{Normal \\ Menisci}
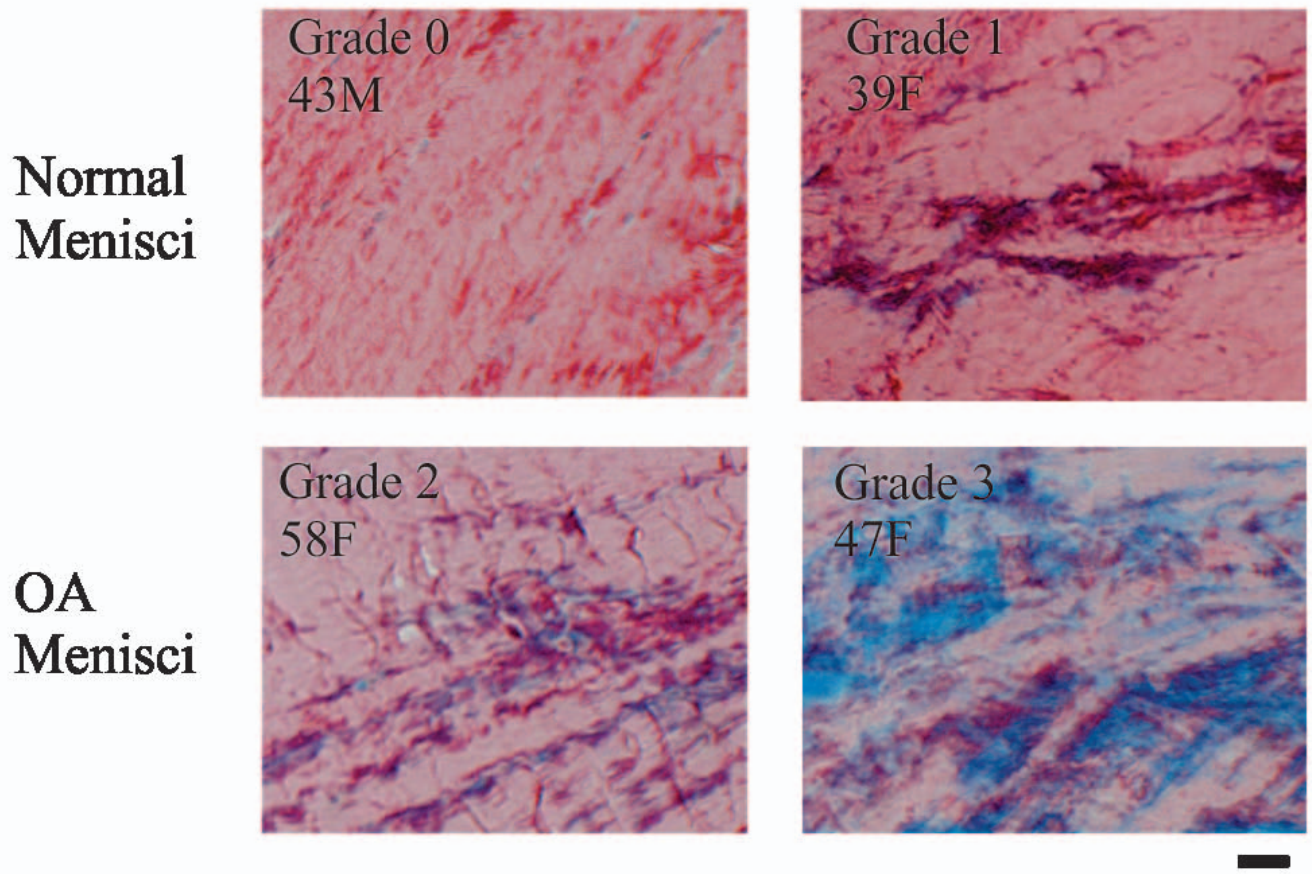

Fig. (2). Picrosirius red staining. Normal menisci (horizontal sections of the central portion of menisci) had strong picrosirius red staining (top photos). In contrast, OA menisci (horizontal sections of the central portion of menisci) had moderate and weak picrosirius red staining mixed with blue counter staining (bottom photos). Note that the decrease of collagen staining occurred inside the OA menisci. Scale bar: 200 $\mu \mathrm{m}$. (Inserts show age in years for male (M) and female (F) subjects in Figs. 2-8).

Table 1. Grades of Picrosirius Red Staining

\begin{tabular}{|l|c|c|c|c|c|c|c|c|c|c|}
\hline & $\begin{array}{c}\text { Normal } \\
\mathbf{1 2 F}\end{array}$ & $\begin{array}{c}\text { Normal } \\
\mathbf{3 9 F}\end{array}$ & $\begin{array}{c}\text { Normal } \\
\mathbf{4 3 M}\end{array}$ & $\begin{array}{c}\text { OA } \\
\mathbf{4 2 M}\end{array}$ & $\begin{array}{c}\text { OA } \\
\mathbf{4 9 F}\end{array}$ & $\begin{array}{c}\text { OA } \\
\mathbf{5 4 F}\end{array}$ & $\begin{array}{c}\text { OA } \\
\mathbf{5 5 M}\end{array}$ & $\begin{array}{c}\text { OA } \\
\mathbf{5 8 F}\end{array}$ & $\begin{array}{c}\text { OA } \\
\mathbf{6 5 F}\end{array}$ & $\begin{array}{c}\text { OA } \\
\mathbf{6 6 F}\end{array}$ \\
\hline \hline Grade $_{\mathrm{T}}$ & 2 & 2 & 2 & 0 & 0 & 1 & 0 & 0 \\
\hline Grade $_{\mathrm{H}}$ & 2 & 2 & 2 & 0 & 0 & 0 & 0 & 0 & 1 \\
\hline
\end{tabular}

Grade $_{\mathrm{T}}-$ Transverse section grade; Grade $\mathrm{L}-$ Horizontal section grade; Ages are listed in years; $\mathrm{M}=$ male, $\mathrm{F}=$ female. The difference of the staining grades between OA group and normal control group was statistically significant $(\mathrm{p}=0.011)$. 


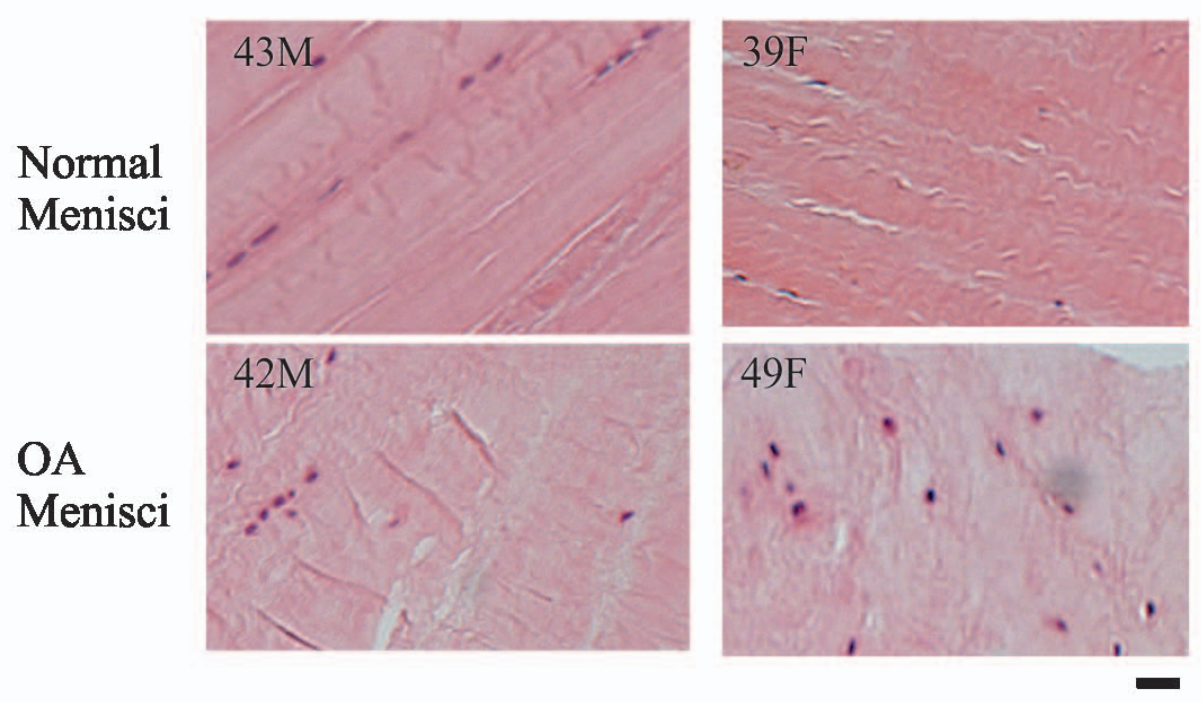

Fig. (3). H\&E staining. Normal menisci (horizontal sections of the central portion of menisci) displayed well organized and compact collagen bundles with meniscal cells lining up between the bundles. In contrast, OA menisci (horizontal sections of the central portion of menisci) displayed less well-organized and less compact collagen networks. In some areas, the meniscal cells were randomly distributed. Scale bar: $200 \mu \mathrm{m}$.

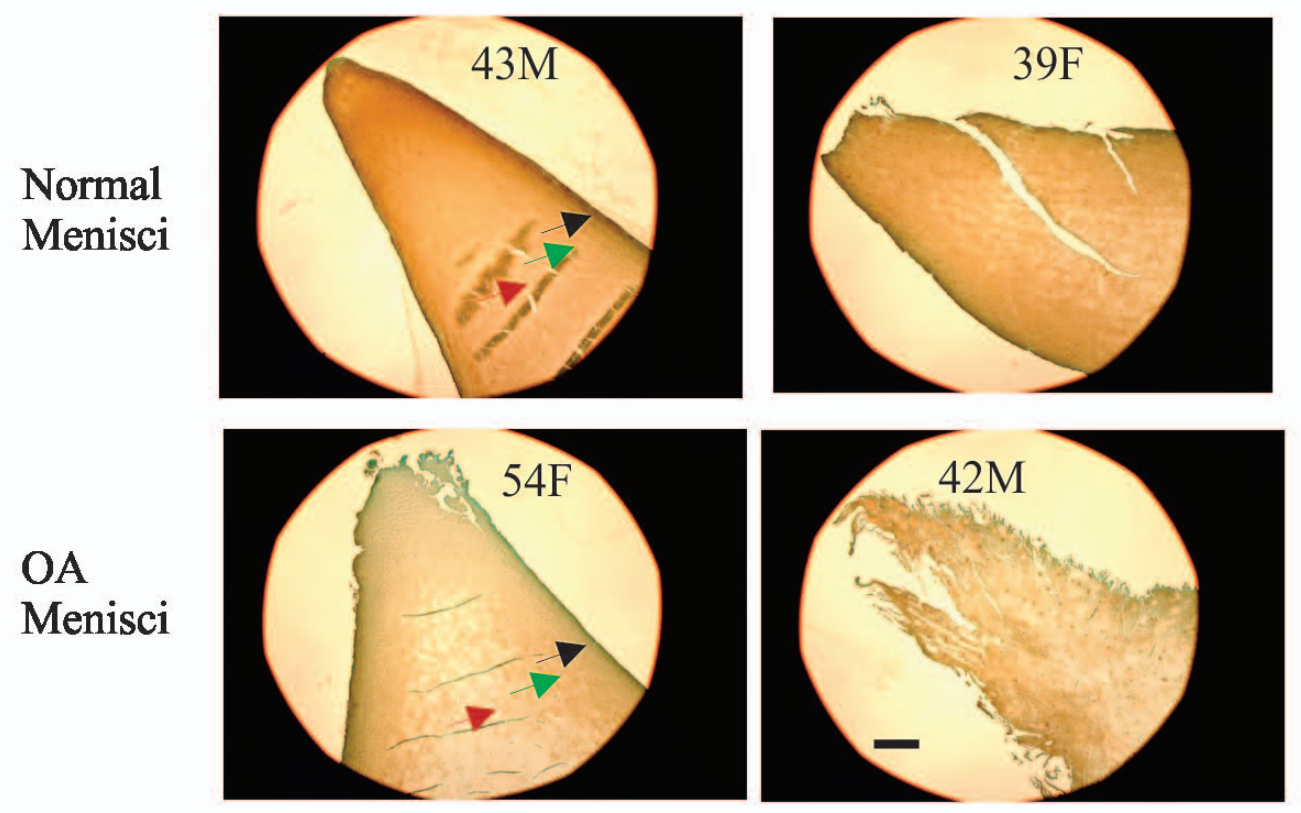

Fig. (4). Immunolocalization of type I collagen. Normal menisci (transverse sections of the inner portion of menisci) showed strong immunolocalization of type I collagen (top photos). Type I collagen staining in the surface zone (black arrow) of normal menisci was greater than that in the middle zone (green arrow) and deep zone (red arrow). Type I collagen staining was decreased in OA menisci compared to normal menisci (compare the bottom photos to the top photos). The reduction of type I collagen staining was much more prominent in the deep zone (the red arrow) than that in the surface zone (the black arrow). Scale bar: $250 \mu \mathrm{m}$.

When compared to normal meniscus, OA meniscus had decreased type I collagen staining throughout the entire meniscus, particularly in the middle zone (green arrow) and deep zone (red arrow), indicating greater severity of type I collagen loss in the middle and deep zones than that in the surface zones (Fig. 4, bottom photos). The grades of immunolocalization of type I collagen, along with the demographic patient information, are listed in Table 2. The decrease of type I collagen staining in OA menisci compared to normal control menisci is statistically significant $(\mathrm{P}=$ $0.021)$.

Representative images of immunolocalization of type II collagen in the inner portion of normal and OA meniscus are shown in Fig. (5). The results indicated that the surface zone (black arrow) of normal meniscus had the highest type II 


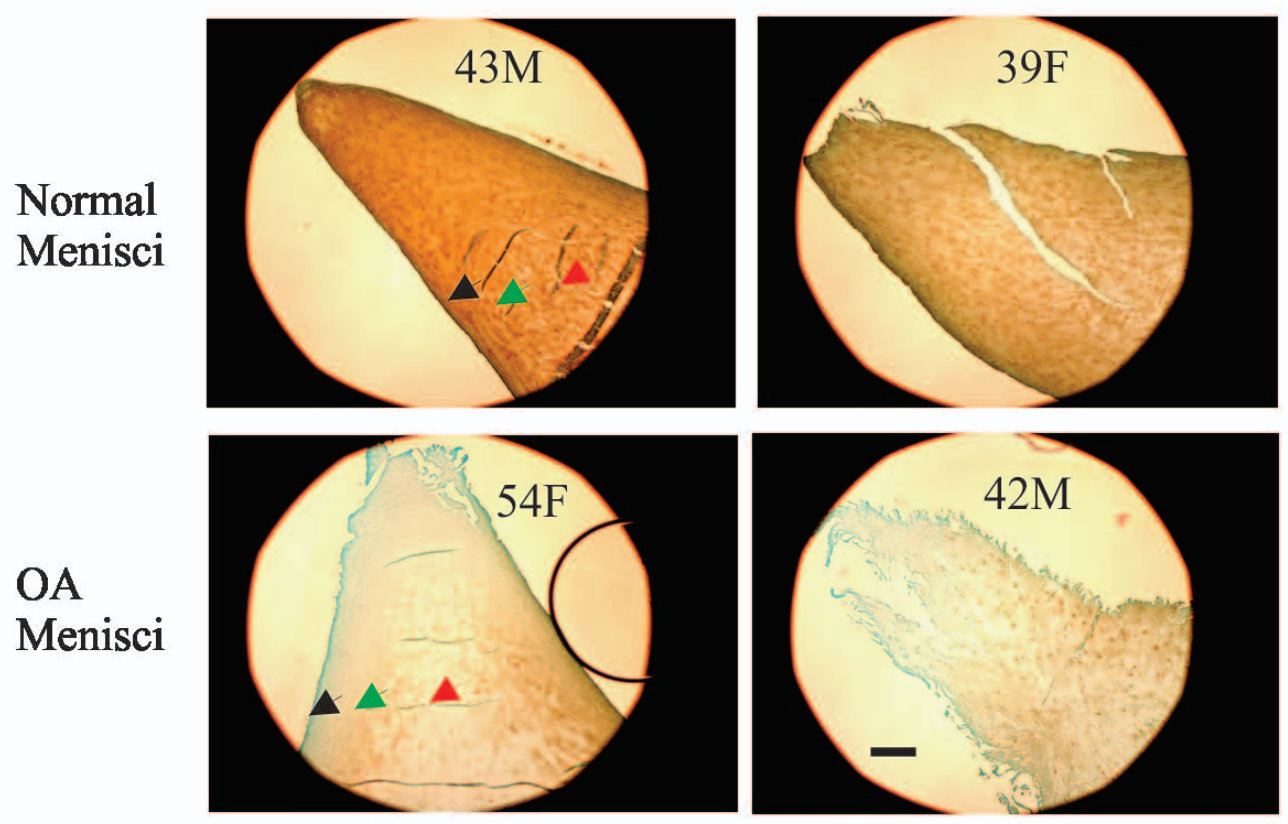

Fig. (5). Immunolocalization of type II collagen. Normal menisci (transverse sections of the inner portion of menisci) showed strong immunolocalization of type II collagen (top photos). Type II collagen staining in the surface zone (black arrow) of normal menisci was greater than that in the middle zone (green arrow) and deep zone (red arrow). Type II collagen staining was decreased in OA menisci compared to normal menisci (compare the bottom photos to the top photos). The reduction of type II collagen staining was prominent in all the three zones. Scale bar: $250 \mu \mathrm{m}$.

Table 2. Grades of Type I Collagen and Type II Collagen Immunolocalization

\begin{tabular}{|c|c|c|c|c|c|c|c|c|c|c|c|}
\hline & $\begin{array}{c}\text { Normal } \\
43 \mathrm{M}\end{array}$ & $\begin{array}{c}\text { Normal } \\
39 F\end{array}$ & $\begin{array}{c}\text { Normal } \\
12 F\end{array}$ & $\begin{array}{c}\text { OA } \\
42 M\end{array}$ & $\begin{array}{l}\text { OA } \\
49 F\end{array}$ & $\begin{array}{l}\text { OA } \\
54 \mathrm{~F}\end{array}$ & $\begin{array}{c}\text { OA } \\
55 M\end{array}$ & $\begin{array}{l}\text { OA } \\
58 \mathrm{~F}\end{array}$ & $\begin{array}{l}\text { OA } \\
65 F\end{array}$ & $\begin{array}{l}\text { OA } \\
66 \mathrm{~F}\end{array}$ & $\begin{array}{l}\text { OA } \\
70 F\end{array}$ \\
\hline \multicolumn{12}{|c|}{ Type I Collagen } \\
\hline Grade $_{I}$ & 3 & 2 & 1 & 0 & 0 & 0 & 1 & 0 & 0 & 0 & 1 \\
\hline \multicolumn{12}{|c|}{ Type II Collagen } \\
\hline Grade $_{\mathrm{I}}$ & 4 & 3 & 2 & 0 & 0 & 0 & 1 & 1 & 0 & 0 & 1 \\
\hline
\end{tabular}

Grade $_{\mathrm{S}}-$ grade of type I collagen in the surface zone; $\mathrm{Grade}_{\mathrm{I}}-$ grade of type I collagen in the deep zone; Ages are listed in years; $\mathrm{M}=$ male, $\mathrm{F}=$ female. The difference of immunolocalization of type I collagen grades between the OA group and the normal control group was statistically significant $(\mathrm{p}=0.021)$. The difference of immunostaining of type II collagen grades between the OA group and the normal control group was statistically significant $(\mathrm{p}=0.018)$.

collagen content, and that the type II collagen content was decreased gradually from the surface zone (black arrow) to the middle zone (green arrow) and the deep zone (red arrow) (Fig. 5, top photos). Similar to type I collagen, type II collagen content was decreased in OA meniscus compared to normal meniscus (Fig. 5, bottom photos). However, different from type I collagen, the decrease of type II collagen content was prominent across all the three zones. The grades of the immunolocalization of type II collagen, along with the demographic patient information, are listed in Table 2.

\section{Safranin-O and Alcian Blue Staining}

Representative images of safranin-O staining are shown in Fig. (6). The safranin-O staining in OA meniscus was significantly stronger than that in normal menisci (Fig. 6A). The increase of safranin-O staining was more prominent in the deep zone (red arrow) than that in the surface zone (black arrow) (Fig. 6B). The grades are listed in Table 3.

Representative images of alcian blue staining are shown in Fig. (7) and the grades of alcian blue staining are listed in Table 3. Consistent with safranin-O staining, alcian blue staining was significantly stronger in the OA menisci compared to the normal menisci (Fig. 7A). This increased staining was seen throughout the OA meniscus and appeared more prominent in the deep zone (red arrow) than that in the surface zone (black arrow) (Fig. 7B). We also performed toluidine blue staining. The results are consistent with that of safranin-O staining and alcian blue staining (Data not shown).

\section{Immunolocalization of Aggrecan}

In previous study, we found that the expression of aggrecan was higher in OA meniscal cell when compared to 
$\mathbf{A}$
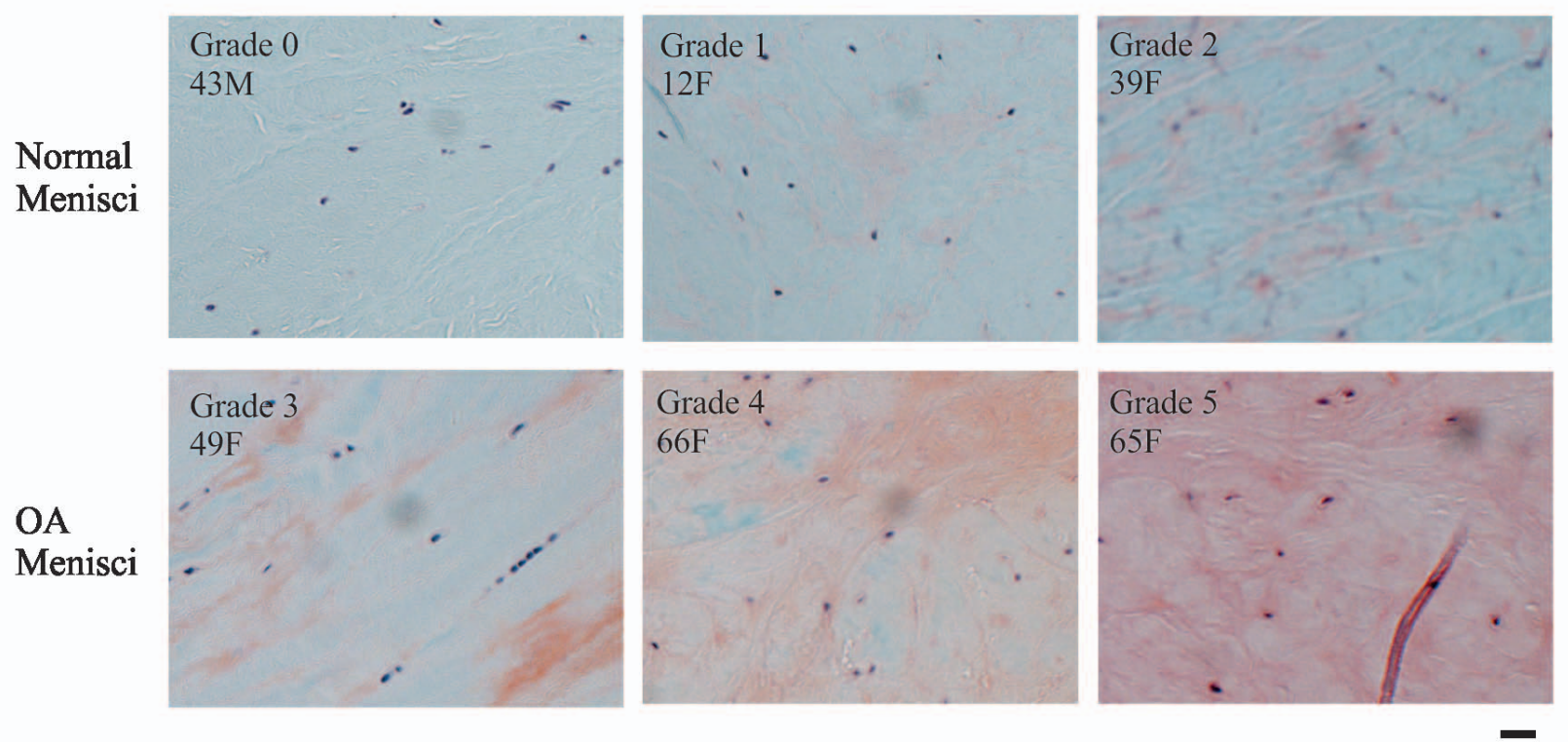

B

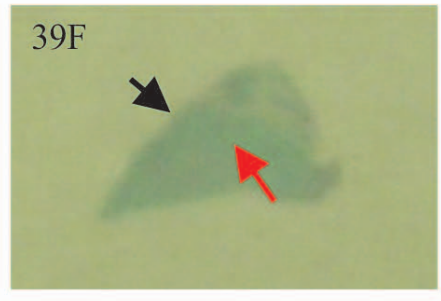

Normal Meniscus

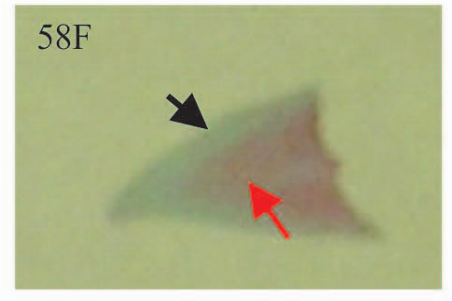

OA Meniscus

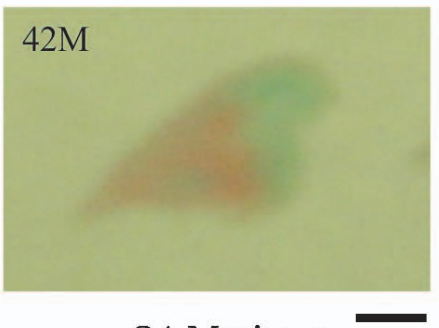

OA Meniscus

Fig. (6). Representative images of safranin-O staining. (A) Normal menisci (horizontal sections of the central portion of menisci) displayed minimal or weak orange-red staining (top photos). In contrast, OA menisci (horizontal sections of the central portion of menisci) displayed moderate to very strong orange-red staining (bottom photos). Scale bar: $200 \mu \mathrm{m}$. (B) Normal menisci (transverse sections of the entire meniscus) displayed minimal orange-red staining. OA menisci (transverse sections of the entire meniscus) displayed moderate to strong orange-red staining. The increase of proteoglycan staining in OA menisci compared to normal menisci was more prominent in the middle and deep zones than that in the surface zone. Scale bar: $2.5 \mathrm{~mm}$.

normal meniscal cells [12]. To further investigate this finding, we examined aggrecan content in the $\mathrm{OA}$ and normal menisci. Aggrecan content was much higher in OA menisci when compared to normal menisci (Fig. 8). The increase of aggrecan staining appeared more prominent in the deep zone than in the surface zone of OA menisci (not shown), indicating active aggrecan synthesis and turn-over inside the OA menisci (Fig. 8). The grades of the immunolocalization of aggrecan along with the demographic patient information are listed in Table 4.

\section{DISCUSSION}

Our study demonstrate that the surface zone of normal menisci has the highest type I and type II collagen contents, and that the deep zone has the lowest, suggesting that in normal menisci the surface zone has the highest tensile strength. Our findings also demonstrate that type I collagen co-localizes with type II collagen in meniscus, which is consistent with a previous report [16].

It is the first time as we know that collagen content is found to decrease not only in the surface zone but also inside the OA menisci. The decrease of type II collagen content in the surface zone is severe. This decrease is likely due to a combination of similar mechanical factors and type-II collagen-degrading factors within the synovial fluid that cause type II collagen degeneration in the surface zone of OA articular cartilage. Interestingly, the decrease of type II collagen content in the middle and deep zones of OA meniscus is also prominent. It suggests that type-II collagendegrading enzymes are also activated inside the OA menisci. The decrease of type I collagen content in all three zones is severe; however, it appeared much more severe in the middle and deep zones than in the surface zone. The distinctive spatial distributions of type I and type II collagen content in 


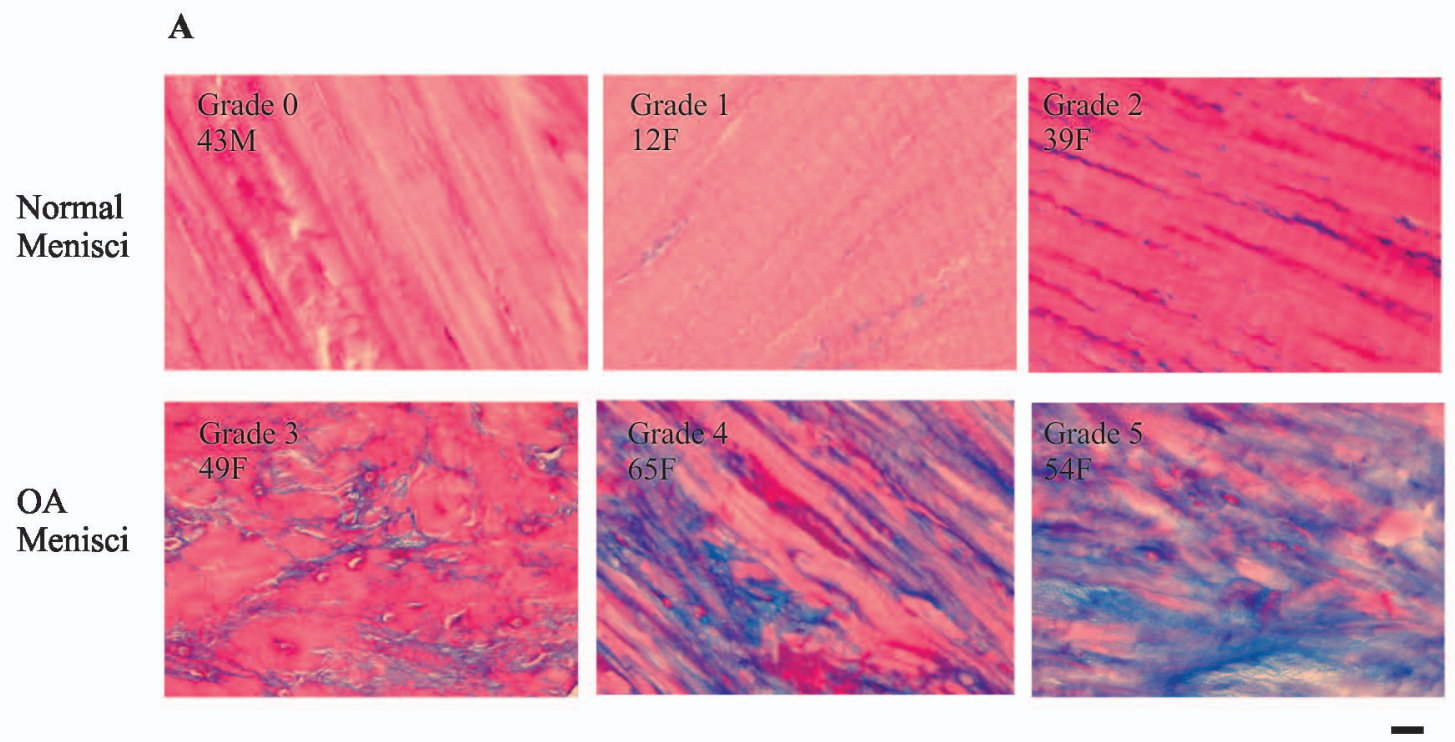

$\mathbf{B}$

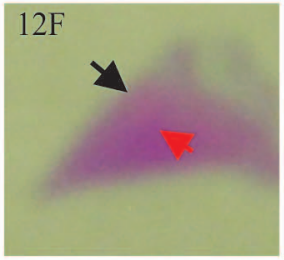

Normal Menisci

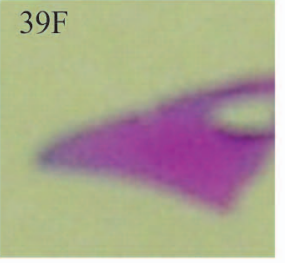

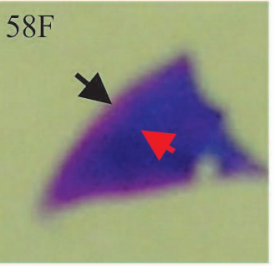

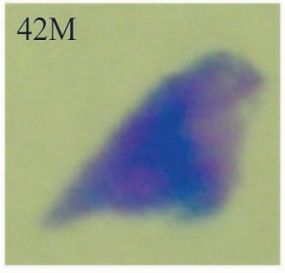

OA Menisci

Fig. (7). Representative images of alcian blue staining. (A) Normal menisci (horizontal sections of the central portion of menisci) displayed minimal or very weak blue staining (top photos). In contrast, OA menisci (horizontal sections of the central portion of menisci) displayed moderate to strong blue staining (bottom photos). Scale bar: $200 \mu \mathrm{m}$. (B) Normal menisci (transverse sections of the entire meniscus) displayed minimal blue staining. OA menisci (transverse sections of the entire meniscus) displayed moderate to strong blue staining. The increase of proteoglycan staining in OA menisci compared to normal menisci was more prominent in the middle and deep zones than that in the surface zone. Scale bar: $2.5 \mathrm{~mm}$.

Table 3. Grades of Safranin-O and Alcian Blue Staining

\begin{tabular}{|c|c|c|c|c|c|c|c|c|c|c|c|}
\hline & $\begin{array}{c}\text { Normal } \\
12 F\end{array}$ & $\begin{array}{c}\text { Normal } \\
\text { 39F }\end{array}$ & $\begin{array}{c}\text { Normal } \\
43 \mathrm{M}\end{array}$ & $\begin{array}{c}\text { OA } \\
42 M\end{array}$ & $\begin{array}{c}\text { OA } \\
49 F\end{array}$ & $\begin{array}{l}\text { OA } \\
54 F\end{array}$ & $\begin{array}{c}\text { OA } \\
55 \mathrm{M}\end{array}$ & $\begin{array}{l}\text { OA } \\
58 F\end{array}$ & $\begin{array}{l}\text { OA } \\
65 F\end{array}$ & $\begin{array}{l}\text { OA } \\
66 F\end{array}$ & $\begin{array}{l}\text { OA } \\
70 F\end{array}$ \\
\hline \multicolumn{12}{|c|}{ Safranin-O } \\
\hline $\operatorname{Grade}_{\mathrm{T}}$ & 0 & 2 & 0 & 3 & 3 & 5 & 4 & 2 & 5 & 4 & 3 \\
\hline Grade $_{\mathrm{H}}$ & 2 & 2 & 0 & 3 & 3 & 5 & 4 & 3 & 5 & 5 & 4 \\
\hline \multicolumn{12}{|c|}{ Alcian Blue } \\
\hline Grade $_{\mathrm{T}}$ & 0 & 1 & 0 & 3 & 4 & 4 & 3 & 3 & 5 & 4 & 4 \\
\hline Grade $_{\mathrm{H}}$ & 1 & 2 & 0 & 3 & 3 & 5 & 4 & 3 & 5 & 4 & 5 \\
\hline
\end{tabular}

$\mathrm{Grade}_{\mathrm{T}}-$ Transverse section grade; Grade $\mathrm{H}_{\mathrm{H}}$ - Horizontal section grade; Ages are listed in years; $\mathrm{M}=$ male, $\mathrm{F}=$ female. The difference of safranin-O staining grades between OA group and normal control group was statistically significant $(\mathrm{p}=0.018)$. The difference of alcian blue staining grades between OA group and normal control group was statistically significant $(\mathrm{p}=0.014)$

OA meniscus compared to normal meniscus suggest that type I collagen-degrading enzymatic activity is much higher in the middle and deep zones than in the surface zone. It also suggests that type II collagen-degrading enzymatic activity is higher across all three zones of OA meniscus compared to the normal meniscus.

Safranin-O staining indicated that proteoglycan content increased in OA menisci when compared to normal menisci. 


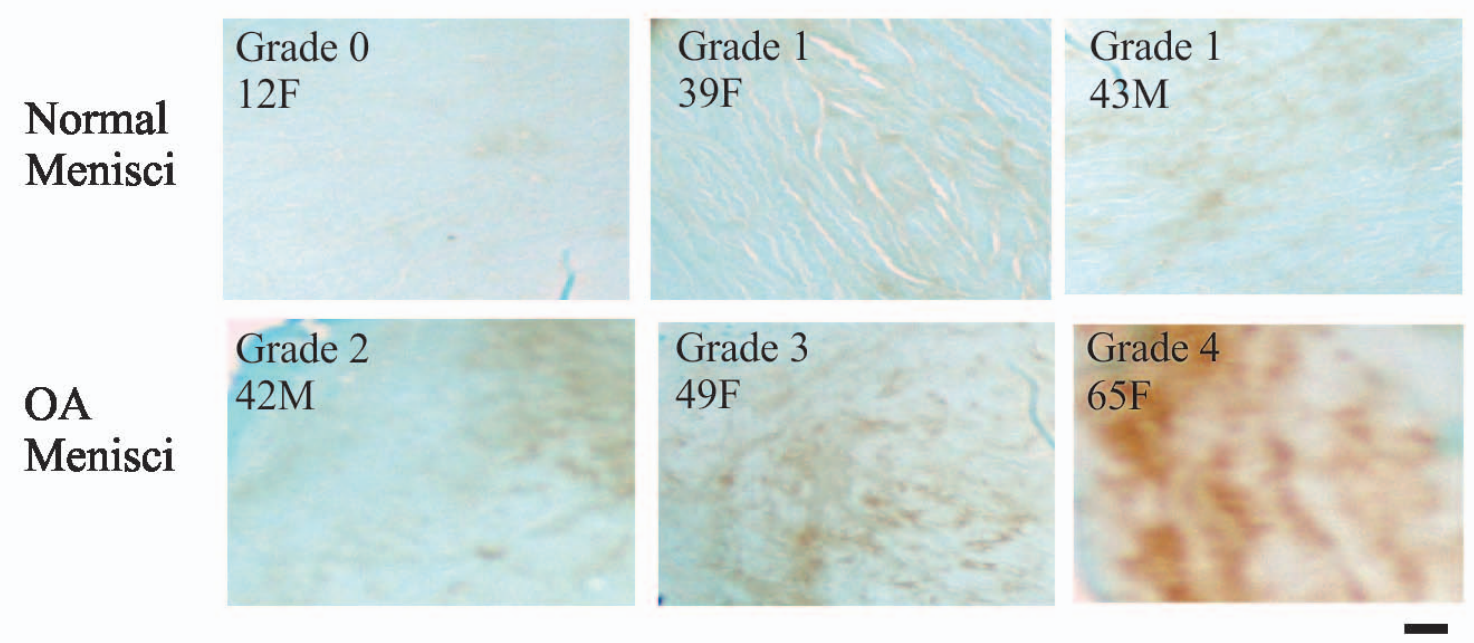

Fig. (8). Immunolocalization of aggrecan. Normal menisci (horizontal sections of the central portion of menisci) showed minimal or weak immunolocalization of aggrecan (top photos). In contrast, OA menisci (horizontal sections of the central portion of menisci) showed moderate to strong immunolocalization of aggrecan (bottom photos). Scale bar: $200 \mu \mathrm{m}$.

Table 4. Grades of Aggrecan Immunolocalization

\begin{tabular}{|c|c|c|c|c|c|c|c|c|c|c|c|}
\hline & $\begin{array}{c}\text { Normal } \\
12 F\end{array}$ & $\begin{array}{c}\text { Normal } \\
\text { 39F }\end{array}$ & $\begin{array}{c}\text { Normal } \\
43 \mathrm{M}\end{array}$ & $\begin{array}{c}\text { OA } \\
42 M\end{array}$ & $\begin{array}{l}\text { OA } \\
49 F\end{array}$ & $\begin{array}{l}\text { OA } \\
54 \mathrm{~F}\end{array}$ & $\begin{array}{c}\text { OA } \\
55 M\end{array}$ & $\begin{array}{l}\text { OA } \\
58 F\end{array}$ & $\begin{array}{l}\text { OA } \\
65 F\end{array}$ & $\begin{array}{l}\text { OA } \\
66 F\end{array}$ & $\begin{array}{l}\text { OA } \\
70 F\end{array}$ \\
\hline Grade $_{\mathrm{T}}$ & 0 & 1 & 1 & 2 & 3 & 2 & 1 & 2 & 3 & 2 & 3 \\
\hline Grade $_{\mathrm{H}}$ & 0 & 0 & 1 & 2 & 3 & 3 & 3 & 4 & 4 & 2 & 2 \\
\hline
\end{tabular}

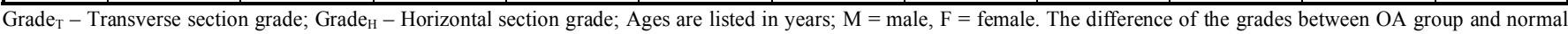
control group was statistically significant $(\mathrm{p}=0.013)$.

Initially, we were surprised by this finding because proteoglycan content decreased in human OA articular cartilage [13-15]. Findings from study of proteoglycan contents using animal OA models were inconsistent. For example, Videman et al., reported that proteoglycan content increased in menisci after induction of OA with limb immobilization in rabbit [17]. Djurasovic et al., reported that proteoglycan content decreased in menisci after induction of OA with limb immobilization in mature beagles [18]. Adams et al., reported that proteoglycan content decreased in menisci during the first three months but increased gradually during the following months after induction of OA with anterior cruciate ligament transection in beagles [19].

Peters and Smillie reported that in patients with meniscal lesions proteoglycan content increased in sections of menisci with degenerative tears [20]. Herwig et al., reported that in patients with meniscal lesions proteoglycan content $(\mu \mathrm{g} / \mathrm{mg}$ dry weight) in menisci increased in relation to the severity of meniscal degeneration [21]. In studies of human meniscal specimens derived from patients with OA, Ghosh et al., found that the proteoglycan content in the degenerative areas of OA menisci, but not in the degenerative areas of rheumatoid arthritis menisci, was increased when compared to normal control menisci [22]. Our findings reported in this study are clearly consistent with these early studies of human meniscal specimens.
Taken together, our findings indicate that meniscal degeneration and cartilage degeneration in $\mathrm{OA}$ follow

different pathways. In OA cartilage both severe collagen loss and proteoglycan loss occur, indicating that both collagendegrading enzymes and proteoglycan-degrading enzymes are actively involved in the degenerative process. In contrast, in OA menisci severe collagen but not proteoglycan loss occurs, indicating that collagen-degrading enzymes are much more actively involved in the degenerative process than proteoglycan-degrading enzymes. The development of structure-modifying drugs that targets meniscal degeneration for OA therapy, therefore, should focus on molecules that inhibit collagen-degrading enzymes. Our findings also indicate that meniscal degeneration in OA may be much more severe than we originally thought because early microscopic studies of OA menisci can not detect intrameniscal degeneration. In addition, it indicates that OA may start inside the meniscus as suggested by a number of MRI studies, which demonstrate that intrameniscal degeneration is a common feature in OA patients [23-26].

\section{CONCLUSION}

In summary, severe loss of collagens and intrameniscal degeneration are hallmarks of $\mathrm{OA}$ menisci and that extracellular matrix degeneration occurred in OA menisci may follow a pathway different from that in OA articular cartilage. These findings are not only important for a better 
understanding of the disease process but also important for the development of novel structure-modifying drugs for OA therapy.

\section{ACKNOWLEDGEMENT}

This study is supported in part by a CharlotteMecklenburg Education and Research Foundation Grant and a Mecklenburg County Medical Society Smith Arthritis Fund Grant (to YS).

\section{CONFLICT OF INTEREST}

Declared none.

\section{ABBREVIATIONS}

$$
\begin{aligned}
& \mathrm{H \& E}=\text { Hematoxylin and eosin staining } \\
& \mathrm{MRI}=\text { Magnetic resonance imaging } \\
& \mathrm{OA}=\text { Osteoarthritis }
\end{aligned}
$$

\section{REFERENCE}

[1] Noble J, Hamblen DL. The pathology of the degenerate meniscus lesion. J Bone Joint Surg Br 1975; 57: 180-6.

[2] Englund M, Guermazi A, Gale D, et al. Incidental meniscal findings on knee MRI in middle-aged and elderly persons. N Engl $\mathbf{J}$ Med 2008; 359: 1108-15.

[3] Chan WP, Lang P, Stevens MP, et al. Osteoarthritis of the knee: comparison of radiography, CT, and MR imaging to assess extent and severity. Am J Roentgenol 1991; 157: 799-806.

[4] Bennett LD, Buckland-Wright JC. Meniscal and articular cartilage changes in knee osteoarthritis: a cross-sectional double-contrast macroradiographic study. Rheumatology 2002; 41: 917-23.

[5] Englund M. Meniscal tear--a feature of osteoarthritis. Acta Orthop Scand 2004; 75: 1-45

[6] Englund M, Guermazi A, Roemer FW, et al. Meniscal tear in knees without surgery and the development of radiographic osteoarthritis among middle-aged and elderly persons: the Multicenter Osteoarthritis Study. Arthritis Rheum 2009; 60: 831-839.

[7] Fukuta S, Kuge A, Korai F. Clinical significance of meniscal abnormalities on magnetic resonance imaging in an older population. Knee 2009; 16: 187-90.

[8] Crema MD, Guermazi A, Li L, et al. The association of prevalent medial meniscal pathology with cartilage loss in the medial tibiofemoral compartment over a 2-year period. Osteoarthritis Cartilage 2010; 18: 336-43

[9] Kai B, Mann SA, King C, Forster BB. Integrity of articular cartilage on T2 mapping associated with meniscal signal change. Eur J Radiol 2011; 79: 421-7.

[10] Kan A, Oshida M, Oshida S, Imada M, Nakagawa T, Okinaga S. Anatomical significance of a posterior horn of medial meniscus: the relationship between its radial tear and cartilage degradation of joint surface. Sports Med Arthrosc Rehabil Ther Technol 2010; 2: 1.
[11] Sun Y, Mauerhan DR, Honeycutt PR, et al. Calcium deposition in osteoarthritic menisci and meniscal cell culture. Arthritis Res Ther 2010; 12: R56.

[12] Sun Y, Mauerhan DR, Honeycutt PR, et al. Analysis of meniscal degeneration and meniscal gene expression. BMC Musculoskelet Disord 2010; 11: 19.

[13] Squires GR, Okouneff S, Ionescu M, Poole AR. The pathobiology of focal lesion development in aging human articular cartilage and molecular matrix changes characteristic of osteoarthritis. Arthritis Rheum 2003; 48: 1261-70.

[14] Lahm A, Mrosek E, Spank H, et al. Changes in content and synthesis of collagen types and proteoglycans in osteoarthritis of the knee joint and comparison of quantitative analysis with Photoshop-based image analysis. Arch Orthop Trauma Surg 2010; 130: 557-64.

[15] Saarakkala S, Julkunen P, Kiviranta P, Makitalo J, Jurvelin JS, Korhonen RK. Depth-wise progression of osteoarthritis in human articular cartilage: investigation of composition, structure and biomechanics. Osteoarthritis Cartilage 2010; 18: 73-81.

[16] Kambic HE, McDevitt CA. Spatial organization of types I and II collagen in the canine meniscus. J Orthop Res 2005; 23: 142-9.

[17] Videman T, Eronen I, Friman C, Langenskiold A. Glycosaminoglycan metabolism of the medial meniscus, the medial collateral ligament and the hip joint capsule in experimental osteoarthritis caused by immobilization of the rabbit knee. Acta Orthop Scand 1979; 50: 465-70.

[18] Djurasovic M, Aldridge JW, Grumbles R, Rosenwasser MP Howell D, Ratcliffe A. Knee joint immobilization decreases aggrecan gene expression in the meniscus. Am J Sports Med 1998; 26; 460-6.

[19] Adams ME, Billingham ME, Muir H. The glycosaminoglycans in menisci in experimental and natural osteoarthritis. Arthritis Rheum 1983; 26: 69-76.

[20] Peters TJ, Smillie IS. Studies on the chemical composition of the menisci of the knee joint with special reference to the horizontal cleavage lesion. Clin Orthop Relat Res 1972; 86: 245-52.

[21] Herwig J, Egner E, Buddecke E. Chemical changes of human knee joint menisci in various stages of degeneration. Ann Rheum Dis 1984; 43: 635-40.

[22] Ghosh P, Ingman AM, Taylor TK. Variations in collagen, noncollagenous proteins, and hexosamine in menisci derived from osteoarthritic and rheumatoid arthritic knee joints. J Rheumatol 1975; $2: 100-7$

[23] Bhattacharyya T, Gale D, Dewire P, et al. The clinical importance of meniscal tears demonstrated by magnetic resonance imaging in osteoarthritis of the knee. J Bone Joint Surg Am 2003; 85-A: 4-9.

[24] Berthiaume MJ, Raynauld JP, Martel-Pelletier J, et al. Meniscal tear and extrusion are strongly associated with progression of symptomatic knee osteoarthritis as assessed by quantitative magnetic resonance imaging. Ann Rheum Dis 2005; 64: 556-63.

[25] Hunter DJ, Zhang YQ, Niu JB, et al. The association of meniscal pathologic changes with cartilage loss in symptomatic knee osteoarthritis. Arthritis Rheum 2006; 54: 795-801.

[26] Roemer FW, Zhang Y, Niu J, et al. Tibiofemoral joint osteoarthritis: risk factors for MR-depicted fast cartilage loss over a 30 -month period in the multicenter osteoarthritis study. Radiology 2009; 252: 772-80

This is an open access article licensed under the terms of the Creative Commons Attribution Non-Commercial License (http://creativecommons.org/licenses/by-nc/ 3.0/) which permits unrestricted, non-commercial use, distribution and reproduction in any medium, provided the work is properly cited. 